\title{
A Study of music analysis method under the trend of modern reconstruction of Chinese national music--Taking the 2 nd movement of Tan Dun's MAP as an example
}

\author{
Ming-qing Liu \\ College of music, Neijiang Normal University, Neijiang, China \\ 330762966@qq.com
}

Keywords: Chinese national music, Reconstruction, Music analysis method, Tan Dun, Blowing leaf.

\begin{abstract}
The reconstruction of Chinese national music is the main means to inherit and protect Chinese traditional folk music, and it is also the main trend of Chinese traditional music development. As the theoretical basis of music creation and music performance, music analysis plays a significant role in the development of national music. Therefore, based on the characteristics of Chinese Traditional music culture, this paper explores the music analysis method under the reconstruction of Chinese national music.
\end{abstract}

\section{Introduction}

The modern reconstruction is an important means of inheriting and developing Chinese folk music, and it is also the mainstream trend of the development of Chinese music culture under the concept of coexistence of diverse cultures. In recent years, Chinese musicians have used Chinese folk music and cultural materials to continuously practice and create a large number of representative modern works. Mr. Tan Dun, an outstanding composer living in the United States, has created a large number of modern musical works based on the folk music. Among them, the Map - to find the disappearing Genlai (ten diaries in western Hunan) is a great works combining the folk music and the Western orchestral music, and also a model of modern reconstruction of traditional Chinese folk music. Tan Dun retained these Chinese native music resources as much as possible through modern technology means $^{[1]}$. The second movement Blowing A Leaf presents a scene that a couple of young Tujjia lovers express their love to each other by blowing a leaf to create music, vivid and interesting.

The music analysis is a means of exploring and analyzing works in depth from multiple levels, in order to comprehend works and summarize laws, and it is necessary for music creation and music performance. Affected by the western music form system, the music analysis method in our country is mainly the western music form system. This makes Chinese traditional music itself ignored, and it is hard to reflect the characteristics of Chinese traditional music. China's traditional music is different from Western music, so the analyzing of the traditional Chinese music should be conducted with our "mother tongue" analysis logic of music form ${ }^{[2]}$. Based on this, I attempt to explore the analysis methods suitable for the musical works under the integration and inheritance of Chinese folk music from the characteristics of Chinese traditional music. The second movement Blowing A Leaf of the works of Map is relatively short, but presents very clear mood, contents and images. With it as an example, this article explores the basic music analysis method, with the purpose to providing the reference for the reconstruction of Chinese folk music.

\section{Analysis on the Creative Thinking and Concept of Blowing a Leaf}

The creative thinking and concept are the basis for analyzing and comprehend a works. They show the overall idea and decide the presentation form of a works The second movement Blowing a Leaf of the works of Map mainly uses orchestral music to imitate Tujia men and women to express their 
feelings with a leave, presenting the harmony between human and nature. The whole band presents the nature. Some of the instruments imitate the sound made by leaves, combined with the multimedia, so that the music has a strong fusion and imaging effect. The movement is relatively short, but presents the scenes of playing, feeling exchanging and love expressing in the nature.

\section{Analysis on the Sound Materials of Blowing a Leaf}

The sound material analysis is the focus of modern music analysis, mainly involving the production of sound prototype, combination and change of sound materials. For the modern reconstructed works with Chinese traditional folk music as the material, the prototype of sound material as well as Chinese traditional sound and pitch are the main motivation of the whole works. So I think, the analysis of the sound prototype material is very important.

The prototype material of Blowing A Leaf is the pitch of the leaf performed by the multimedia, which is is bright, cheerful and playful. The performance of music is mainly through the use of Western orchestral music to imitate this pitch. The imitation of wood wind instruments, the conversation between the wood wind instruments and the multimedia, as well as the native pitch of the violoncello are all the imitation and performance of the original pitch. While the other instruments in the Western orchestra exist as a backdrop by imitating the sounds of nature.

\section{Analysis on the Creative Technique of Blowing a Leaf}

Corresponding to the technique analysis of Western traditional music which involves harmony, polyphony and musical form, the modern music analysis mainly involves the production way, combination means and changes of sound through the use of $f$ modern technologies. The imitation of various sounds in nature and the expression of feelings between men and women via the sounds in the nature itself are the main creative techniques of the works of Blowing a Leaf.

The works begins with a clarinet imitating the music by blowing a leaf, and uses the percussion music as a background, presenting a scene that a breeze is blowing through and the leaves of trees in a forest are rustling. Immediately after this scene, the string music is introduced, and the percussion music becomes strong, and the trill of the piccolo among the wood wind instruments brings the first small climax. The introduction of the leave sound into the multimedia directly blends the most traditional expression style of the folk music with the modern orchestral music, forming a very vivid picture together with the scenes depicted by the other instruments in the orchestra. And then, the sound of the violoncello enters, its narrative pitch is presented three times. The violoncello and the violin respectively imitate the sound of leave, as they are talking to each other. Finally, the sound of the harp joins in, so the whole music culminates and ends. In a word, Blowing A Leaf is performed using the techniques of imitating the sounds in the nature and achieving the change and repetition of pitch in the folk music.

\section{Analysis on the Aesthetic Significance of Blowing a Leaf}

As a model for the modern reconstructed works of Chinese folk music, and rooted on the international stage, the works of Blowing A Leaf not only shows a unique creative thinking, novel sound material, and super instruments arrangement means, but also meets the aesthetic ideas of Chinese people and even the people across the world. Chinese people always pursue artistic conception. They view pictures as beauty, and express their feelings through pictures. On the one hand, Blowing A Leaf depicts a very vivid picture; and on the other hand, it expresses the delicate emotions deep into the heart by the combination of the clarinet, the violoncello, the violin and the leaf. Therefore, this works is of great significance to the development of Chinese traditional folk music. 


\section{Conclusion}

The total time length of Blowing A Leaf is 4min. With the main pitch of blowing a leaf in the multimedia as the original sound and the expressing of subtle feelings of men and women as the main idea, the works uses the modern techniques to combine the music made by various instruments in the Western orchestra with Chinese traditional folk music, forming a very artistic works. For the analysis of these works, I think that we should first analyze the composition of Chinese traditional folk music, including the composition of sound, the composition of pitch and the connotation of their thoughts. Secondly, we should analyze the process of modernization of Chinese folk orchestral music, that is, analyzing the creative techniques of the works and the composition of the band; and finally, we should analyze the aesthetic significance and value of the works.

\section{References}

[1] X.K. Song. Inheriting the Past and Forging ahead into the Future-Creation of The Map by Tan Dun and a Probe into Ethnic Original Music.J. Journal of Minzu University of China (Philosophy and Social Sciences Edition) . Vol.40, No.6(2013)149-152.

[2] F. Liu. Multi-integration and Reconstruction--A Description of 'The Academic Symposium on reflection and Construction of Chinese national musicology’. J. Music Space. No.5(2016)7-12 